\title{
Thermochemistry of heteroatomic compounds: analysis and calculation of thermodynamic functions of organic compounds of V-VII groups of Mendeleev's Periodic table
}

\section{Vitaly Vitalevich Ovchinnikov \\ Department of General Chemistry and Ecology, Tupolev Kazan National Researching Technical University, Tatarstan, Russia}

Email address:

chem_vvo@mail.ru

\section{To cite this article:}

Vitaly Vitalevich Ovchinnikov. Thermochemistry of Heteroatomic Compounds: Analysis and Calculation of Thermodynamic Functions of Organic Compounds of V-VII Groups of Mendeleev's Periodic Table. American Journal of Physical Chemistry.

Vol. 2, No. 4, 2013, pp. 60-71. doi: 10.11648/j.ajpc.20130204.11

\begin{abstract}
The heat of vaporization $\left(\Delta_{\mathrm{vap}} H^{\mathrm{o}}\right)$, all thermodynamic functions $\Delta_{\mathrm{c}, \mathrm{f}} G^{\mathrm{o}}, \Delta_{\mathrm{c}, \mathrm{f}} H^{\mathrm{o}}, S_{\mathrm{o}}, \Delta_{\mathrm{c}} S_{\text {cond }}^{\mathrm{o}}$ and heat capacity $\left(C_{\mathrm{p}}\right)$ of organic compounds of V-VII groups of Mendeleev's Periodic Table can be well characterized with the number of valence electrons $N$ without taking into account the number $(h)$ of lone electrons pairs $(g)$ of them in equation $\Delta_{\text {vap,c,f,s }} \Psi^{\circ}=i \pm f^{*}(N-$ $h g$ ). The stoichiometric coefficients $i$ and $f$ reflect partially a various spatial structure of all investigated compounds. The free energy and entropy of combustion and formation are calculated only for oxygen, sulfur and partly fluorine compounds. For this reason their analysis causes the certain difficulties. It is possible to note, that $f$ coefficients in the given above equation for free energies of combustion are very close to such coefficients for the heat of combustion processes. Also it has been found that calculated thermodynamic functions of ethers and sulfides are in good interdependence from each other: $\Delta_{\mathrm{c}} H^{0}-\Delta_{\mathrm{c}} S_{\text {cond }}^{\mathrm{o}}, \Delta_{\mathrm{f}} H^{0}-S_{\text {cond }}^{\mathrm{o}}, S_{\text {cond }}^{\mathrm{o}}-C_{p}$. The 74 equations of mentioned type have been created for processes of vaporization, combustion, formation, entropic transformations and heat capacity.
\end{abstract}

Keywords: Free Energy of Combustion, Free Energy of Formation, Heat of Combustion Heat of Formation, Entropy, Heat Capacity, Organic Compounds

\section{Introduction}

In the present communication we continue to analyze and calculate the changes of thermodynamic functions $\Delta \Psi^{\circ}$ $\left(\Delta G^{\mathrm{o}}, \Delta H^{\mathrm{o}}, \Delta S^{\mathrm{o}}\right)$, characterizing the processes of vaporization, combustion and formation of the condensed and gaseous organic compounds and their heat capacities $\left(C_{p}\right)$ of V-VII groups of Mendeleev's Periodic Table.

Previously we have repeatedly shown, that not only the heat of combustion of organometallic compounds of I-IV groups of Periodic Table [1], organic [2, 3] and biochemical compounds, such as different carbohydrates [4] and amino acids [5] in the dependence from the number of their valence electrons $N$ are.

We hope to investigate same interrelations (Equation 1, in which $i$ and $f$ are stoichiometric coefficients) in the rows of mentioned above of heteroatomic compounds now

$$
\Delta_{\text {vap,c, }, \mathrm{s}, \mathrm{s}} \Psi^{\mathrm{o}}=i \pm f^{*}(N-h g)
$$

We wished to remind, that in accordance to Kharasch conception "The electronic conception of valence and heats of combustion of organic compounds" [6] the number $(h)$ of lone electron pairs $(g=2)$ should not take part in the combustion process, apparently. We have chosen the $\mathrm{IV}^{\text {th }}$ group of Periodic Table (compounds of carbon and below) as a base point since in these compounds are not present a lone electron pairs $(h g=0)$ [7]. According to this position the parameter $h$ is equal to 1 and $h g=2$, for compounds of $\mathrm{V}^{\text {th }}$ group the parameter $h$ is equal to 2 and $h g=4$ for $\mathrm{VI}^{\text {th }}$ group and the parameter $h$ is equal to 3 and $h g=6$ for VII $^{\text {th }}$ group of Periodic system [7].

\section{Results and Discussion}

\subsection{Organic Compounds of $V^{\text {th }}$ Group (Trialkyl (Aryl)Amines and-Phosphines)}

The analysis of thermodynamic functions of nitrogen (IIX) and phosphorus (X-XIV, Table 1) compounds should be begun with consideration of their heats of vaporization $\left(\Delta_{\text {vap }} H^{\circ}\right)$. This thermochemical parameter quantitatively 
depends from a number of valence electrons $N$, from which is subtracted one $(h)$ lone electron pair, hence contribution $h g$ is equally 2 (Eqs. 2 and 3, they are presented in Table 2 also)

$$
\begin{gathered}
\Delta_{\text {vap }} H^{\mathrm{o}}=(8.3 \pm 10.4)+(0.7 \pm 0.1)(N-h g) \quad r 0.960, S_{\mathrm{o}} 11.2, n 5 \text { (compounds I-III, VI, IX) } \\
\Delta_{\text {vap }} H^{\mathrm{o}}=(-8.4 \pm 1.4)+(1.4 \pm 0.2)(N-h g) \quad r 0.991, S_{\mathrm{o}} 9.1, n 3 \text { (compounds X-XII) }
\end{gathered}
$$

Processes of combustion of the same nitrogen and phosphorus organic compounds can be presented by the

$$
\begin{aligned}
& \mathrm{R}_{3} \mathrm{~N}+n \mathrm{O}_{2} \rightarrow x \mathrm{CO}_{2} \text { (gas) }+y \mathrm{H}_{2} \mathrm{O}\left(\text { liq) }+z \mathrm{~N}_{2}\right. \text { (gas) } \\
& \mathrm{R}_{3} \mathrm{P}+n \mathrm{O}_{2} \rightarrow x \mathrm{CO}_{2} \text { (gas) }+y \mathrm{H}_{2} \mathrm{O}(\text { liq })+z \mathrm{H}_{3} \mathrm{PO}_{4} \text { (sol) }
\end{aligned}
$$

in which $n, x, y, z$ are stoichiometric coefficients, and $\mathrm{R}$ are Alkyl, Aryl.

The analysis of the combustion heats of these two groups

$$
\begin{aligned}
& \Delta_{\mathrm{c}} H^{\mathrm{o}}=(-21.8 \pm 29.5)-(108.8 \pm 0.4)(N-h g) \quad r 0.999, S_{\mathrm{o}} 39.5, n 9 \text { (compounds I-IX) } \\
& \Delta_{\mathrm{c}} H^{\mathrm{o}}=(-854.8 \pm 45.4)-(107.0 \pm 0.5)(N-h g) r 0.999, S_{\mathrm{o}} 52.8, n 5 \text { (compounds X-XIV) }
\end{aligned}
$$

It is necessary to note, that stoichiometric coefficients $f$ in these equations are very close to coefficient $-109.0 \mathrm{~kJ}$ $\mathrm{mol}^{-1}$ electron ${ }^{-1}$ for the similar equations on combustion of alkanes [3], nitro compounds [2], carbohydrates [4] and amino acids [5].

The heats of formation of nitrogen and phosphorus compounds in condensed and gas phases are characterized by good dependences (8-11, Table 2) also.

$$
\begin{aligned}
& \Delta_{\mathrm{f}} H_{\text {cond }}^{\mathrm{o}}=(52.8 \pm 23.9)-(4.3 \pm 0.2)(N-h g) \quad r 0.991, S_{\mathrm{o}} 21.2, n 9 \text { (compounds I-IX) } \\
& \Delta_{\mathrm{f}} H_{\text {gas }}^{\mathrm{o}}=(50.4 \pm 4.0)-(3.5 \pm 0.04)(N-h g) \quad r 0.999, S_{\mathrm{o}} 4.2, n 4 \text { (compounds I-III, VI) } \\
& \Delta_{\mathrm{f}} H_{\text {cond }}^{\mathrm{o}}=(-191.5 \pm 58.2)+(3.9 \pm 0.6)(N-h g) r 0.964, S_{\mathrm{o}} 67.7, n 5 \text { (compounds X-XIV) } \\
& \Delta_{\mathrm{f}} H_{\text {gas }}^{\mathrm{o}}=(-284.3 \pm 4.0)+(7.1 \pm 1.2)(N-h g) \quad r 0.985, S_{\mathrm{o}} 60.4, n 3 \text { (compounds X-XII) }
\end{aligned}
$$

The values entropy of formation and heat capacity are known for compounds (I and VI, Table 1) only. Nevertheless the first of them has allowed to calculate the entropy of combustion for trimethylamine (I) into the

$$
\Delta_{\mathrm{c}} S_{\text {cond }}^{\mathrm{o}} \mathrm{P}=\sum n_{\mathrm{i}} S_{\text {products }}^{\mathrm{o}} \mathrm{P} \sum \sum n_{\mathrm{j}} S_{\text {reactants }}^{\mathrm{o}}
$$

In turn, the value of $\Delta_{c} S_{\text {cond }}^{o}$ Phas enabled to calculate the energies of combustion and formation (Table 1) in condensed state for amine (I) through the Eqs. (13 and 14)

$$
\begin{aligned}
& \Delta_{\mathrm{c}} G^{\mathrm{o}}=\Delta_{\mathrm{c}} H^{\mathrm{o}}-298.15 \cdot \Delta_{\mathrm{c}} S^{\mathrm{o}} \\
& \Delta_{\mathrm{f}} G^{\mathrm{o}}=\Delta_{\mathrm{f}} H^{\mathrm{o}}-298.15 \cdot S^{\mathrm{o}}
\end{aligned}
$$

\subsection{Organic Compounds of $V^{\text {th }}$ Group $x$ (Trialkyl(Aryl)Arsines, -Stibines and - Bismuthines)}

accordance with the scheme (4) and equation (12). The required values of entropy of formation for molecules $\mathrm{CO}_{2}$, $\mathrm{H}_{2} \mathrm{O}$ and $\mathrm{N}_{2}$ (213.8, 70.1 and $191.5 \mathrm{~J} \mathrm{~mol}^{-1} \mathrm{~K}^{-1}$ correspondingly) were taken in monograph [8].

$$
\text { here } n_{\mathrm{i}} \text { and } n_{\mathrm{j}} \text { are stoichiometric coefficients }
$$

The sequence of the thermodynamic functions research for arsenic, stibium and bismuth compounds remains the same, which has been chosen for the previous compounds: analysis of vaporization, combustion, heats of formation and after this the calculation of entropy and free energies of combustion and formation.

The consideration of known in the literature values of vaporization enthalpies (XV-XXIII, Table 1) leads us to the receiving of Eqs. (15-17, Table 2)

$$
\begin{array}{ll}
\Delta_{\text {vap }} H^{\mathrm{o}}=(-9.0 \pm 10.8)+(1.5 \pm 0.2)(N-h g) & r 0.992, S_{\mathrm{o}} 9.2, n 3 \text { (compounds XV-XVII) } \\
\Delta_{\text {vap }} H^{\mathrm{o}}=(1.9 \pm 7.8)+(1.2 \pm 0.1)(N-h g) & r 0.993, S_{\mathrm{o}} 6.8, n 3 \text { (compounds XXVIII-XX) } \\
\Delta_{\mathrm{vap}} H^{\mathrm{o}}=(5.3 \pm 9.8)+(1.2 \pm 0.2)(N-h g) & r 0.990, S_{\mathrm{o}} 2.8, n 3 \text { (compounds XXI-XXIII) }
\end{array}
$$

The processes of combustion of arsenic, stibium and schemes (18-20) bismuth compounds it is possible to present three following

$$
\begin{aligned}
& \mathrm{R}_{3} \mathrm{As}+n \mathrm{O}_{2} \rightarrow x \mathrm{CO}_{2} \text { (gas) }+y \mathrm{H}_{2} \mathrm{O} \text { (liq) }+z \mathrm{As}_{2} \mathrm{O}_{3} \text { (sol) } \\
& \mathrm{R}_{3} \mathrm{Sb}+n \mathrm{O}_{2} \rightarrow x \mathrm{CO}_{2} \text { (gas) }+y \mathrm{H}_{2} \mathrm{O} \text { (liq) }+z \mathrm{Sb}_{2} \mathrm{O}_{4} \text { (sol) }
\end{aligned}
$$




$$
\mathrm{R}_{3} \mathrm{Bi}+n \mathrm{O}_{2} \rightarrow x \mathrm{CO}_{2} \text { (gas) }+y \mathrm{H}_{2} \mathrm{O} \text { (liq) }+z \mathrm{Bi}_{2} \mathrm{O}_{3} \text { (sol) }
$$

in which $n, x, y, z$ are stoichiometric coefficients, and $\mathrm{R}$ is Alk, Ar.

The use of literary values ([Reference 9]?] of the heats of combustion and formation in condensed and gas phase for

$$
\begin{aligned}
& \Delta_{\mathrm{c}} H^{\mathrm{o}}=(-385.8 \pm 70.7)-(110.3 \pm 1.2)(N-h g) r 0.999, S_{\mathrm{o}} 59.6, n 3 \text { (compounds XV-XVII) } \\
& \Delta_{\mathrm{f}} H_{\text {cond }}^{\mathrm{o}}=(-198.0 \pm 77.2)+(6.6 \pm 1.3)(N-h g) r 0.979, S_{\mathrm{o}} 65.1, n 3 \text { (compounds XV-XVII) } \\
& \Delta_{\mathrm{f}} H_{\text {gas }}^{\mathrm{o}}=(-207.9 \pm 87.5)+(8.0 \pm 1.5)(N-h g) r 0.982, S_{\mathrm{o}} 73.7, n 3 \text { (compounds XV-XVII) } \\
& \Delta_{\mathrm{c}} H^{\mathrm{o}}=(-528.6 \pm 34.6)-(107.8 \pm 0.6)(N-h g) \quad r 0.999, S_{\mathrm{o}} 29.2, n 3 \text { (compounds XVIII-XX) } \\
& \Delta_{\mathrm{f}} H_{\text {cond }}^{\mathrm{o}}=(-155.5 \pm 80.0)+(5.3 \pm 1.4)(N-h g) r 0.967, S_{\mathrm{o}} 67.1, n 3 \text { (compounds XVIII-XX) } \\
& \Delta_{\mathrm{f}} H^{\mathrm{o}} \text { gas }=(-152.6 \pm 88.5)+(6.5 \pm 1.5)(N-h g) \quad r 0.973, S_{\mathrm{o}} 74.6, n 3 \text { (compounds XVIII-XX) } \\
& \Delta_{\mathrm{c}} H^{\mathrm{o}}=(-621.4 \pm 113.2)-(106.6 \pm 2.0)(N-h g) r 0.999, S_{\mathrm{o}} 95.4, n 3 \text { (compounds XXI-XXIII) } \\
& \Delta_{\mathrm{f}} H_{\text {cond }}^{\mathrm{o}}=(14.5 \pm 68.5)+(5.0 \pm 1.2)(N-h g) \quad r 0.973, S_{\mathrm{o}} 57.7, n 3 \text { (compounds XXI-XXIII) } \\
& \Delta_{\mathrm{f}} H_{\text {gas }}^{\circ}=(18.6 \pm 77.3)+(6.2 \pm 1.3)(N-h g) \quad r 0.977, S_{\mathrm{o}} 65.2, n 3 \text { (compounds XXI-XXIII) }
\end{aligned}
$$

\begin{tabular}{|c|c|c|c|c|c|c|c|c|c|c|}
\hline \multirow[t]{2}{*}{$\begin{array}{l}\text { No } \\
\text { comp. }\end{array}$} & \multirow[t]{2}{*}{$\begin{array}{l}\text { Title of Compound, } \\
\text { formula, } N \text {-gh }\end{array}$} & \multicolumn{2}{|c|}{ Free energy } & \multicolumn{4}{|c|}{ Enthalpy (heat contributions) } & \multicolumn{3}{|c|}{ Entropy and heat capacity } \\
\hline & & $\begin{array}{l}-\Delta_{\mathrm{c}} \boldsymbol{G}^{\mathbf{0}} \\
(\text { Calc. } \\
\pm 0.5)^{\mathrm{a}}\end{array}$ & $\begin{array}{l}-\Delta_{\mathrm{f}} \boldsymbol{G}^{\mathbf{0}} \\
(\text { Calc. } \\
\pm 0.5)^{\mathrm{b}}\end{array}$ & $-\Delta_{\mathrm{vap}} H^{\mathbf{0}}$ & $-\Delta_{\mathrm{c}} \boldsymbol{H}^{0}$ & $-\Delta_{\mathrm{f}} \boldsymbol{H}^{0}$ & $-\Delta_{\mathrm{f}} \boldsymbol{H}_{\text {gas }}^{0}$ & $S^{\mathbf{0}}$ & $\begin{array}{l}-\Delta_{\mathrm{c}} \boldsymbol{S}^{\mathbf{0}} \\
(\text { Calc. } \\
\pm 0.5)^{\mathrm{e}}\end{array}$ & $C p$ \\
\hline 1 & 2 & 3 & 4 & 5 & 6 & 7 & 8 & 9 & 10 & 11 \\
\hline \multicolumn{11}{|c|}{ Nitrogen compounds } \\
\hline I & $\begin{array}{l}\text { Trimethylamine, } \\
\mathrm{C}_{3} \mathrm{H}_{9} \mathrm{~N}, 22\end{array}$ & $\begin{array}{l}2410.6 \\
\pm 12.0\end{array}$ & $\begin{array}{l}104.7 \\
\pm 0.5\end{array}$ & $\begin{array}{l}22.0^{[10]} \\
\pm 0.1\end{array}$ & $\begin{array}{l}2484.0^{[11]} \\
\pm 12.4\end{array}$ & $\begin{array}{l}45.7^{[10]} \\
\pm 0.7\end{array}$ & $\begin{array}{l}23.7^{[10]} \\
\pm 0.7^{[0}\end{array}$ & ${ }_{[12]}^{197.8}$ & $\begin{array}{l}246.1 \\
\pm 1.2\end{array}$ & $132.5^{[12]}$ \\
\hline II & Triethylamine, $\mathrm{C}_{6} \mathrm{H}_{15} \mathrm{~N}, 40$ & & & $\begin{array}{l}34.9^{[13]} \\
\pm 0.1\end{array}$ & $\begin{array}{l}4377.1^{[14]} \\
\pm 0.5\end{array}$ & $\begin{array}{l}127.8 \\
{[14]} \\
\pm 0.5\end{array}$ & $\begin{array}{l}92.9^{[14]} \\
\pm 0.5\end{array}$ & & & \\
\hline III & $\begin{array}{l}\text { Tri-n-ropylamine, } \\
\mathrm{C}_{9} \mathrm{H}_{21} \mathrm{~N}, 58\end{array}$ & & & $\begin{array}{l}46.1^{[13]} \\
\pm 0.1\end{array}$ & $\begin{array}{l}6335.7^{[14]} \\
\pm 0.8\end{array}$ & $\begin{array}{l}206.9 \\
{[14]} \\
\pm 0.8\end{array}$ & $\begin{array}{l}160.8 \\
{[14]} \\
\pm 0.8\end{array}$ & & & \\
\hline IV & $\begin{array}{l}\text { Tri-n-butylamine, } \\
\mathrm{C}_{12} \mathrm{H}_{27} \mathrm{~N}, 76\end{array}$ & & & & $\begin{array}{l}8299.2^{[14]} \\
\pm 1.1\end{array}$ & $\begin{array}{l}281.8 \\
{[14]} \\
\pm 1.1\end{array}$ & & & & \\
\hline V & $\begin{array}{l}\text { Tri-n-hexylamine, } \\
\mathrm{C}_{18} \mathrm{H}_{39} \mathrm{~N}, 112\end{array}$ & & & & $\begin{array}{l}12223.8 \\
{[14]} \\
\pm 1.5\end{array}$ & $\begin{array}{l}433.0 \\
{[14]} \\
\pm 1.8\end{array}$ & & & & \\
\hline VI & $\begin{array}{l}\text { Tri-n-octylamine, } \\
\mathrm{C}_{24} \mathrm{H}_{51} \mathrm{~N}, 148\end{array}$ & & & $\begin{array}{l}110.0 \\
{[15]} \\
\pm 15.0\end{array}$ & $\begin{array}{l}16147.9 \\
{[14] \quad \pm 2.1}\end{array}$ & $\begin{array}{l}585.0 \\
{[14]} \\
\pm 2.6\end{array}$ & $\begin{array}{l}474.8 \\
{[14]}\end{array}$ & & & $750.8^{[15]}$ \\
\hline VII & $\begin{array}{l}\text { Tri-n-nonylamine, } \\
\mathrm{C}_{27} \mathrm{H}_{57} \mathrm{~N}, 166\end{array}$ & & & & $\begin{array}{l}18109.3^{[9]} \\
\pm 1.7\end{array}$ & $\begin{array}{l}661.6^{[9]} \\
\pm 2.3\end{array}$ & & & & \\
\hline VIII & $\begin{array}{l}\text { Tri-n-decylamine, } \\
\mathrm{C}_{30} \mathrm{H}_{63} \mathrm{~N}, 184\end{array}$ & & & & $\begin{array}{l}20070.9 \\
{[14] \quad \pm 3.1}\end{array}$ & $\begin{array}{l}738.0 \\
{[14]} \\
\pm 3.6\end{array}$ & & & & \\
\hline IX & $\begin{array}{l}\text { Triphenylamine, } \mathrm{C}_{18} \mathrm{H}_{15} \mathrm{~N} \text {, } \\
88\end{array}$ & & & $\begin{array}{l}92.0^{[16]} \\
\pm 3.0^{-}\end{array}$ & $\begin{array}{l}9462.0^{[16]} \\
\pm 3.0\end{array}$ & $\begin{array}{l}235.0 \\
{[16]} \\
\pm 3.0\end{array}$ & $\begin{array}{l}-327.0 \\
{[16]} \\
\pm 4.2\end{array}$ & & & $297.5^{[17]}$ \\
\hline Phosph & compounds & & & & & & & & & \\
\hline
\end{tabular}

The known data (Which?[9]] on heat capacity for arsenic compounds (XV-XVII) have allowed to receive the

equation (30), which reflect the interdependence between the $C_{p}$ values and the number of valence electrons $(N-h g)$

$$
C_{p}=(117.2 \pm 32.5)+(2.3 \pm 0.6)(N-h g) \quad r 0.973, S_{0} 27.4, n 3 \text { (compounds XV-XVII) }
$$

The entropy and free energies of combustion and trimethylarsine (XV) only (Table 1). formation in condensed state are calculated for

Table1. Thermodynamic functions $\left(\mathrm{kJ} \mathrm{mol}^{-1}, \mathrm{~J} \mathrm{~mol}^{-1} \mathrm{~K}^{-1}\right)$ of organic compounds at $P 101 \mathrm{kPa}$ and $298.15 \mathrm{~K}$; all compounds are in condensed state 


\begin{tabular}{|c|c|c|c|c|c|c|c|c|c|c|}
\hline \multirow{2}{*}{$\begin{array}{l}\text { No } \\
\text { comp. } \\
X\end{array}$} & \multirow{2}{*}{$\begin{array}{l}\text { Title of Compound, } \\
\text { formula, } \boldsymbol{N} \text { - } \boldsymbol{g} \boldsymbol{h} \\
\text { Trimethylphosphine, } \\
\mathrm{C}_{3} \mathrm{H}_{9} \mathrm{P}, 22\end{array}$} & \multicolumn{2}{|c|}{ Free energy } & \multicolumn{4}{|c|}{ Enthalpy (heat contributions) } & \multicolumn{3}{|c|}{ Entropy and heat capacity } \\
\hline & & & & \multirow{3}{*}{$\begin{array}{l}28.5^{[18]} \\
\pm 0.9^{[18]} \\
41.1^{[18]} \\
\pm 2.0 \\
118.3 \\
{[18]} \\
\pm 0.6\end{array}$} & \multirow{3}{*}{$\begin{array}{l}3194.9^{[9]} \\
\pm 4.6 \\
5176.0^{[9]} \\
\pm 12.5 \\
10299.0^{[9]} \\
\pm 12.5\end{array}$} & \multirow{3}{*}{$\begin{array}{l}122.2^{[9]} \\
\pm 4.6^{[9]} \\
89.1^{[9]} \\
\pm 12.5 \\
-232.2 \\
{[9]}\end{array}$} & \multirow{3}{*}{$\begin{array}{l}94.1^{[9]} \\
\pm 5.0^{[9]} \\
49.4^{[9]} \\
\pm 13.0 \\
-350.5^{\mathrm{c}} \\
\pm 19.8^{\circ}\end{array}$} & & & \\
\hline XI & $\begin{array}{l}\text { Triethylphosphine, } \mathrm{C}_{6} \mathrm{H}_{15} \mathrm{P} \text {, } \\
40\end{array}$ & & & & & & & & & \\
\hline XII & $\begin{array}{l}\text { Triphenylphosphine, } \\
\mathrm{C}_{18} \mathrm{H}_{15} \mathrm{P}, 88\end{array}$ & & & & & & & & & \\
\hline XIII & $\begin{array}{l}\text { Pentaphenylphosphole, } \\
\mathrm{C}_{34} \mathrm{H}_{25} \mathrm{P}, 162\end{array}$ & & & & $\begin{array}{l}18198.3^{[9]} \\
\pm 28.4\end{array}$ & $\begin{array}{l}-395.8 \\
{[9]} \\
\pm 28.4\end{array}$ & & & & \\
\hline XIV & $\begin{array}{l}\text { 9-Phenyl-9- } \\
\text { phosphafluorene, } \mathrm{C}_{18} \mathrm{H}_{13} \mathrm{P} \text {, } \\
86\end{array}$ & & & & $\begin{array}{l}9982.2^{[9]} \\
\pm 16.7\end{array}$ & $\begin{array}{l}-190.8 \\
{[9]} \\
\pm 16.7\end{array}$ & & & & \\
\hline Arsenic c & mpounds & & & & & & & & & \\
\hline XV & $\begin{array}{l}\text { Trimethylarsine, } \\
\mathrm{C}_{3} \mathrm{H}_{9} \mathrm{As}, 22\end{array}$ & $\begin{array}{l}2481.4 \\
\pm 12.4\end{array}$ & $\begin{array}{l}91.2 \\
\pm 0.5\end{array}$ & $\begin{array}{l}28.9^{[9]} \\
\pm 1.2\end{array}$ & $\begin{array}{l}2779.0^{[9]} \\
\pm 10.0\end{array}$ & $\begin{array}{l}16.3^{[9]} \\
\pm 10.0\end{array}$ & $\begin{array}{l}-11.7^{[9]} \\
\pm 10.5\end{array}$ & $\underset{[19]}{251.3}$ & $\begin{array}{l}998.1 \\
\pm 5.0\end{array}$ & $154.0^{[19]}$ \\
\hline XVI & $\begin{array}{l}\text { Triethylarsine, } \\
\mathrm{C}_{6} \mathrm{H}_{15} \mathrm{As}, 40\end{array}$ & & & $\begin{array}{l}43.1^{[9]} \\
\pm 4.2\end{array}$ & $\begin{array}{l}4846.3^{[9]} \\
\pm 16.7\end{array}$ & $\begin{array}{l}-13.0^{[9]} \\
\pm 16.7\end{array}$ & $\begin{array}{l}-56.1^{[9]} \\
\pm 17.6\end{array}$ & & & $234.3^{[20]}$ \\
\hline XVII & $\begin{array}{l}\text { Triphenylarsine, } \\
\mathrm{C}_{18} \mathrm{H}_{15} \mathrm{As}, 88\end{array}$ & & & $\begin{array}{l}123.6 \\
{[22] \pm 0.6}\end{array}$ & $\begin{array}{l}10082.6^{[9]} \\
\pm 16.7\end{array}$ & $\begin{array}{l}-393.3 \\
{[9]} \\
\pm 16.7\end{array}$ & $\begin{array}{l}-516.9^{\mathrm{c}} \\
\pm 17.3^{\circ}\end{array}$ & & & $321.3^{[21]}$ \\
\hline Stibium c & mpounds & & & & & & & & & \\
\hline XVIII & $\begin{array}{l}\text { Trimethylstibine, } \mathrm{C}_{3} \mathrm{H}_{9} \mathrm{Sb} \text {, } \\
22\end{array}$ & & & $\begin{array}{l}31.4^{[9]} \\
\pm 1.3^{9}\end{array}$ & $\begin{array}{l}2921.0^{[9]} \\
\pm 25.1\end{array}$ & $\begin{array}{l}-0.8^{[9]} \\
\pm 25.1\end{array}$ & $\begin{array}{l}-32.2^{[9]} \\
\pm 25.1\end{array}$ & & & \\
\hline XIX & $\begin{array}{l}\text { Triethylstibine, } \mathrm{C}_{6} \mathrm{H}_{15} \mathrm{Sb} \text {, } \\
40\end{array}$ & & & $\begin{array}{l}43.5^{[9]} \\
\pm 4.2^{[9}\end{array}$ & $\begin{array}{l}4864.3^{[9]} \\
\pm 10.5\end{array}$ & $\begin{array}{l}-5.0^{[9]} \\
\pm 10.5\end{array}$ & $\begin{array}{l}-48.5^{[9]} \\
\pm 10.9\end{array}$ & & & $242.2^{[23]}$ \\
\hline $\mathrm{XX}$ & $\begin{array}{l}\text { Triphenylstibine, } \mathrm{C}_{18} \mathrm{H}_{15} \mathrm{Sb} \text {, } \\
88\end{array}$ & & & $\begin{array}{l}106.3^{[9]} \\
\pm 8.4^{9]}\end{array}$ & $\begin{array}{l}10009.8^{[9]} \\
\pm 16.7\end{array}$ & $\begin{array}{l}-329.3 \\
{[9]} \\
\pm 16.7\end{array}$ & $\begin{array}{l}-435.5 \\
{[9]} \\
\pm 18.8\end{array}$ & & & \\
\hline Bismuth & mpounds & & & & & & & & & \\
\hline XXI & $\begin{array}{l}\text { Trimethylbismuthine, } \\
\mathrm{C}_{3} \mathrm{H}_{9} \mathrm{Bi}, 22\end{array}$ & & & $\begin{array}{l}36.0^{[9]} \\
\pm 1.2\end{array}$ & $\begin{array}{l}2912.1^{[9]} \\
\pm 14.2\end{array}$ & $\begin{array}{l}-158.2 \\
{[9]} \\
\pm 14.2\end{array}$ & $\begin{array}{l}-192.9 \\
{[9]} \\
\pm 14.2\end{array}$ & & & \\
\hline XXII & $\begin{array}{l}\text { Triethylbismuthine, } \\
\mathrm{C}_{6} \mathrm{H}_{15} \mathrm{Bi}, 40\end{array}$ & $\begin{array}{l}4687.9 \\
\pm 23.4\end{array}$ & $\begin{array}{l}-56.9 \\
\pm 0.3\end{array}$ & $\begin{array}{l}46.0^{[9]} \\
\pm 4.2^{[9}\end{array}$ & $\begin{array}{l}4961.4^{[9]} \\
\pm 16.7\end{array}$ & $\begin{array}{l}-169.9 \\
{[9]} \\
\pm 16.7\end{array}$ & $\begin{array}{l}-215.9 \\
{[9]} \\
\pm 17.6\end{array}$ & $\begin{array}{l}379.0 \\
{[24]}\end{array}$ & $\begin{array}{l}917.5 \\
\pm 4.6\end{array}$ & $242.2^{[24]}$ \\
\hline XXIII & $\begin{array}{l}\text { Triphenylbismuthine, } \\
\mathrm{C}_{18} \mathrm{H}_{15} \mathrm{Bi}, 88\end{array}$ & & & $\begin{array}{l}110.9^{[9]} \\
\pm 8.4\end{array}$ & $\begin{array}{l}9983.0^{[9]} \\
\pm 16.7\end{array}$ & $\begin{array}{l}-469.0 \\
{[9]} \\
\pm 16.7\end{array}$ & $\begin{array}{l}-579.9 \\
{[9]} \\
\pm 18.8\end{array}$ & & & $328.4^{[21]}$ \\
\hline Oxygen c & mpounds & & & & & & & & & \\
\hline XXIV & Dimethyl ether, $\mathrm{C}_{2} \mathrm{H}_{6} \mathrm{O}, 12$ & $\begin{array}{l}1423.7 \\
\pm 7.1\end{array}$ & $\begin{array}{l}260.4 \\
\pm 1.3\end{array}$ & $21.5^{[25]}$ & $\begin{array}{l}1460.4^{[26]} \\
\pm 0.46\end{array}$ & & $\begin{array}{l}184.1 \\
{[26]} \\
\pm 0.50\end{array}$ & 146.6 & $\begin{array}{l}124.0 \\
\pm 0.6\end{array}$ & $102.3^{[25]}$ \\
\hline $\mathrm{XXV}$ & Diethyl ether, $\mathrm{C}_{4} \mathrm{H}_{10} \mathrm{O}, 24$ & $\begin{array}{l}2670.6 \\
\pm 13.3\end{array}$ & $\begin{array}{l}346.8 \\
\pm 1.7\end{array}$ & $27.4^{[27]}$ & $\begin{array}{l}2732.1^{[28]} \\
\pm 1.9\end{array}$ & $\begin{array}{l}271.2 \\
{[28]} \\
\pm 1.9\end{array}$ & $\begin{array}{l}252.7 \\
{[29]} \\
\pm 2.0\end{array}$ & $\begin{array}{l}253.5 \\
{[30]}\end{array}$ & $\begin{array}{l}206.4 \\
\pm 1.0\end{array}$ & $172.5^{[30]}$ \\
\hline XXVI & $\begin{array}{l}\text { Di-n-propyl ether, n- } \\
\mathrm{C}_{6} \mathrm{H}_{14} \mathrm{O}, 36\end{array}$ & $\begin{array}{l}3915.0 \\
\pm 19.6\end{array}$ & $\begin{array}{l}367.8 \\
\pm 1.8\end{array}$ & $35.7^{[31]}$ & $\begin{array}{l}4033.1^{[32]} \\
\pm 0.79\end{array}$ & $\begin{array}{l}328.8 \\
{[32]} \\
\pm 0.88\end{array}$ & $\begin{array}{l}293.0 \\
{[32]} \\
\pm 2.0\end{array}$ & $\begin{array}{l}323.9 \\
{[33]}\end{array}$ & $\begin{array}{l}396.3 \\
\pm 2.0\end{array}$ & $221.6^{[33]}$ \\
\hline XXVII & $\begin{array}{l}\text { Di-iso-propyl ether, i- } \\
\mathrm{C}_{6} \mathrm{H}_{14} \mathrm{O}, 36\end{array}$ & $\begin{array}{l}3898.0 \\
\pm 19.5\end{array}$ & $\begin{array}{l}442.3 \\
\pm 2.2\end{array}$ & $32.1^{[31]}$ & $\begin{array}{l}4010.4^{[32]} \\
\pm 1.3\end{array}$ & $\begin{array}{l}351.5 \\
{[32]} \\
\pm 1.4\end{array}$ & $\begin{array}{l}318.0 \\
{[32]} \\
\pm 3.0\end{array}$ & $\begin{array}{l}304.6 \\
{[33]}\end{array}$ & $\begin{array}{l}377.0 \\
\pm 1.9\end{array}$ & $216.1^{[33]}$ \\
\hline XXVIII & $\begin{array}{l}\text { Di-n-Butyl ether, n- } \\
\mathrm{C}_{8} \mathrm{H}_{18} \mathrm{O}, 48\end{array}$ & & & $45.0^{[31]}$ & $\begin{array}{l}5342.6^{[32]} \\
\pm 0.92\end{array}$ & $\begin{array}{l}378.0 \\
{[32]} \\
\pm 1.0\end{array}$ & $\begin{array}{l}334.0 \\
{[32]} \\
\pm 2.0\end{array}$ & & & $278.2^{[34]}$ \\
\hline XXIX & $\begin{array}{l}\text { Di-sec-butyl ether, } \\
\mathrm{s}-\mathrm{C}_{8} \mathrm{H}_{18} \mathrm{O}, 48\end{array}$ & & & $\begin{array}{l}40.6^{[9]} \\
\pm 1.2\end{array}$ & $\begin{array}{l}5319.0^{[9]} \\
\pm 1.0\end{array}$ & $\begin{array}{l}401.5^{[9]} \\
\pm 1.2\end{array}$ & $\begin{array}{l}360.9^{[9]} \\
\pm 1.7\end{array}$ & & & \\
\hline $\mathrm{XXX}$ & $\begin{array}{l}\text { Di-tert-Butyl ether, } \\
\text { t- } \mathrm{C}_{8} \mathrm{H}_{18} \mathrm{O}, 48\end{array}$ & & & $\begin{array}{l}37.6^{[35]} \\
\pm 0.9\end{array}$ & $\begin{array}{l}5322.1^{[36]} \\
\pm 0.61\end{array}$ & $\begin{array}{l}398.4 \\
{[36]} \\
\pm 0.72\end{array}$ & $\begin{array}{l}361.1 \\
{[36]} \\
\pm 0.8\end{array}$ & & & $276.1^{[35]}$ \\
\hline XXXI & $\begin{array}{l}\text { Diphenyl ether, } \mathrm{C}_{12} \mathrm{H}_{10} \mathrm{O} \text {, } \\
56\end{array}$ & $\begin{array}{l}6057.3 \\
\pm 30.3\end{array}$ & $\begin{array}{l}101.8 \\
\pm 0.5\end{array}$ & $\begin{array}{l}66.9^{[37]} \\
\pm 0.3\end{array}$ & $\begin{array}{l}6113.7^{[38]} \\
\pm 3.8\end{array}$ & $\begin{array}{l} \pm 0.12 \\
32.1^{[39]} \\
\pm 0.93\end{array}$ & & $\begin{array}{l}233.9 \\
{[39]}\end{array}$ & $\begin{array}{l}189.2 \\
\pm 0.9\end{array}$ & $216.6^{[39]}$ \\
\hline Sulfur co & pounds & & & & & & & & & \\
\hline XXXII & $\begin{array}{l}\text { Dimethyl sulfide, } \mathrm{C}_{2} \mathrm{H}_{6} \mathrm{~S} \text {, } \\
12\end{array}$ & & & $\begin{array}{l}27.9^{[40]} \\
\pm 0.6\end{array}$ & $\begin{array}{l}2181.5^{[41]} \\
\pm 0.3\end{array}$ & $\begin{array}{l}65.4^{[40]} \\
\pm 1.5\end{array}$ & $\begin{array}{l}37.5^{[40]} \\
\pm 2.0\end{array}$ & & & \\
\hline XXXIII & $\begin{array}{l}\text { Ethylmethyl sulfide, } \\
\mathrm{C}_{2} \mathrm{H}_{5} \mathrm{SCH}_{3}, 18\end{array}$ & & & $\begin{array}{l}32.0^{[42]} \\
\pm 0.1\end{array}$ & $\begin{array}{l}2833.6^{[42]} \\
\pm 1.1\end{array}$ & $\begin{array}{l}92.3^{[42]} \\
\pm 1.1\end{array}$ & $\begin{array}{l}60.3^{[42]} \\
\pm 1.1\end{array}$ & & & \\
\hline XXXIV & Diethyl sulfide, $\mathrm{C}_{4} \mathrm{H}_{10} \mathrm{~S}, 24$ & $\begin{array}{l}3380.6 \\
\pm 16.9\end{array}$ & $\begin{array}{l}199.6 \\
\pm 1.0\end{array}$ & $\begin{array}{l}35.8^{[40]} \\
\pm 0.7\end{array}$ & $\begin{array}{l}3486.1^{[43]} \\
\pm 0.67\end{array}$ & 119.3 & $\begin{array}{l}83.5^{[40]} \\
\pm 2.3\end{array}$ & $\begin{array}{l}269.3 \\
{[44]}\end{array}$ & $\begin{array}{l}353.7 \\
\pm 1.8\end{array}$ & $171.4^{[44]}$ \\
\hline
\end{tabular}




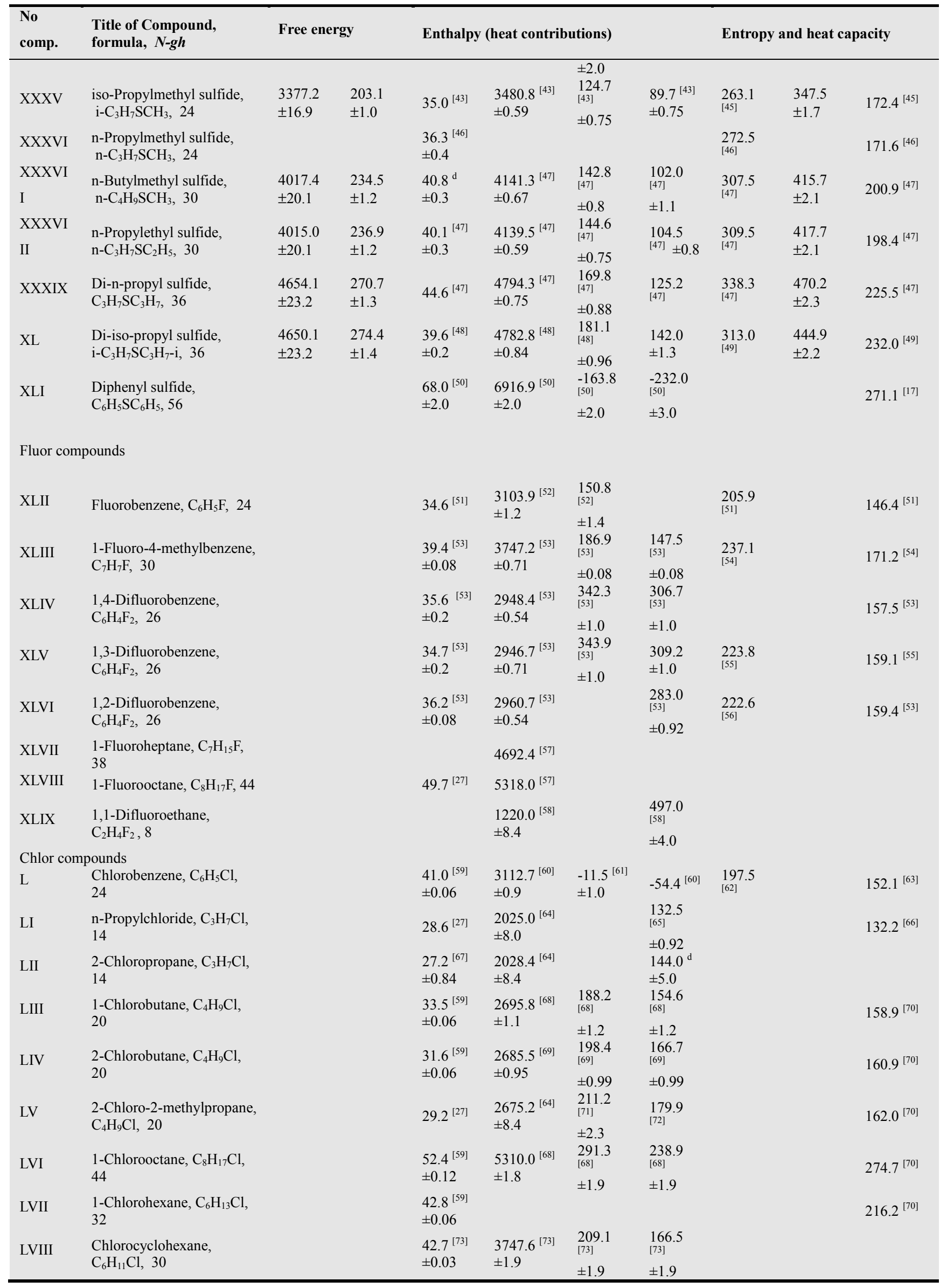




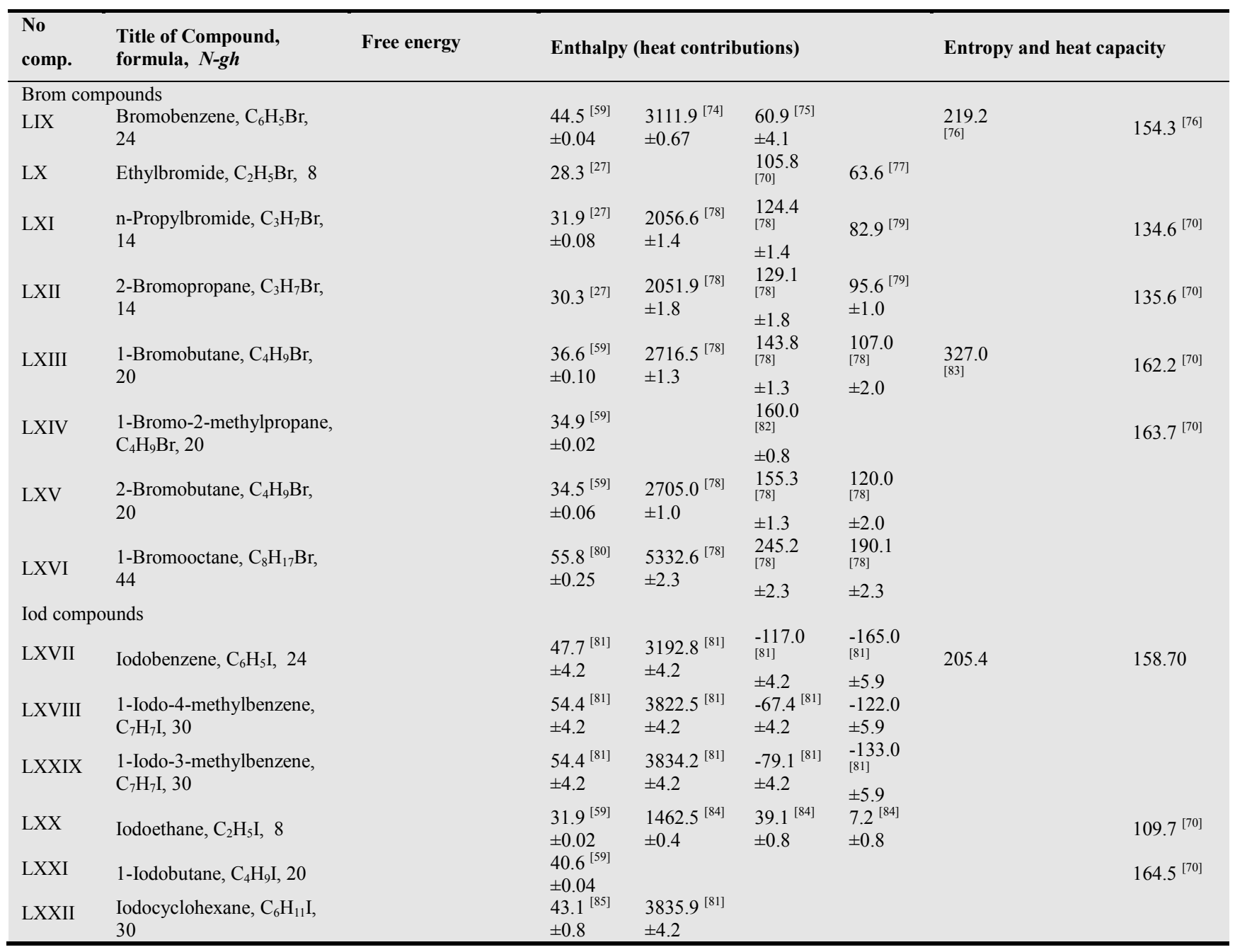

${ }^{\text {a }}$ Calc. through Eq. (13); ${ }^{\mathrm{b}}$ Calc. through Eq. (14); ${ }^{\mathrm{c}}$ Calc. as difference: $\Delta_{\mathrm{f}} \mathrm{H}^{\mathrm{o}}$ cond $-\Delta_{\text {vap }} \mathrm{H}^{\mathrm{o}} ;{ }^{\mathrm{d}}$ Average of 6 values; ${ }^{\mathrm{e}}$ Calc. through Eq. (12).

\subsection{Organic Compounds of VI ${ }^{\text {th }}$ Group (Ethers and Sulfides)}

The thermodynamic data of ethers and sulfides are presented most full among heteroatomic compounds of $\mathrm{VI}^{\text {th }}$ group. There is an opportunity to analyze all thermodynamic functions and heat capacity of compounds
(XXIV-XLI, Table 1). First of all we shall carry out the research of the heats of vaporization of ethers (XXIVXXXI). The received equation (31) shows a good dependence of $\Delta_{\text {vap }} H^{\circ}$ of ethers on the number of valence electrons $(N-g)$. The similar dependence (32) is received for the heats of vaporization of sulfides (XXXII-XLI, Table 2)

Table 2. The parameters of equations $\Delta \Psi^{(o)}\left(\mathrm{kJ} \mathrm{mol}^{-1}\right.$ and $\left.J \mathrm{~mol}^{-1} \mathrm{~K}^{-1}\right)=i \pm f^{*}(\mathrm{~N}$-gh) for vaporization, combustion, formation of organic compounds of $V$ VII groups; $P 101 \mathrm{kPa}$; $\mathrm{T} 298.15 \mathrm{~K}$

\begin{tabular}{|c|c|c|c|c|c|c|}
\hline $\begin{array}{l}\text { The functions } \\
\text { of processes }\end{array}$ & $\begin{array}{l}\text { No Eq. } \\
\text { text }\end{array}$ & $i$ & $f$ & $r$ & $S_{o}$ & Nos. of compounds in Table 1 \\
\hline 1 & 2 & 3 & 4 & 5 & 6 & 7 \\
\hline \multicolumn{7}{|l|}{ Nitrogen compounds } \\
\hline$\Delta_{\text {vap }} H^{\mathrm{o}}$ & 2 & $8.3 \pm 10.4$ & $0.7 \pm 0.1$ & 0.960 & 11.2 & I-III, VI, IX \\
\hline$\Delta_{\mathrm{c}} H^{\mathrm{o}}$ & 6 & $-21.8 \pm 41.6$ & $-108.8 \pm 0.4$ & 0.999 & 59.5 & I-IX \\
\hline$\Delta_{\mathrm{f}} H_{\text {cond }}^{\mathrm{o}}$ & 8 & $57.8 \pm 23.9$ & $-4.3 \pm 0.2$ & 0.991 & 34.1 & I-IX \\
\hline$\Delta_{\mathrm{f}} H_{\text {gas }}^{\mathrm{o}}$ & 9 & $50.4 \pm 4.0$ & $-3.5 \pm 0.04$ & 0.999 & 4.7 & I-III, VI \\
\hline \multicolumn{7}{|c|}{ Phosphorus compounds } \\
\hline$\Delta_{\mathrm{vap}} H^{\mathrm{o}}$ & 3 & $-8.4 \pm 10.8$ & $1.4 \pm 0.2$ & 0.991 & 9.1 & X-XII \\
\hline$\Delta_{\mathrm{c}} H^{\mathrm{o}}$ & 7 & $-854.8 \pm 45.4$ & $-107.0 \pm 0.5$ & 0.999 & 52.8 & X-XIV \\
\hline$\Delta_{\mathrm{f}} H_{\text {cond }}^{\mathrm{o}}$ & 10 & $-191.5 \pm 58.2$ & $3.9 \pm 0.6$ & 0.964 & 67.7 & X-XIV \\
\hline$\Delta_{\mathrm{f}} H_{\text {gas }}^{\mathrm{o}}$ & 11 & $-284.3 \pm 71.7$ & $7.1 \pm 1.2$ & 0.985 & 60.4 & X-XII \\
\hline \multicolumn{7}{|l|}{ Arsenic compounds } \\
\hline$\Delta_{\text {vap }} H^{\circ}$ & 15 & $-9.0 \pm 10.8$ & $1.5 \pm 0.2$ & 0.992 & 9.2 & XV-XVII \\
\hline$\Delta_{\mathrm{c}} H^{\mathrm{o}}$ & 21 & $-385.8 \pm 70.7$ & $-110.3 \pm 1.2$ & 0.999 & 59.6 & XV-XVII \\
\hline$\Delta_{\mathrm{f}} H_{\text {cond }}^{\mathrm{o}}$ & 22 & $-198.0 \pm 77.2$ & $6.6 \pm 1.3$ & 0.979 & 65.1 & XV-XVII \\
\hline$\Delta_{\mathrm{f}} H_{\text {gas }}^{\mathrm{o}}$ & 23 & $-207.9 \pm 87.5$ & $8.0 \pm 1.5$ & 0.982 & 73.7 & XV-XVII \\
\hline
\end{tabular}




\begin{tabular}{|c|c|c|c|c|c|c|}
\hline $\begin{array}{l}\text { The functions } \\
\text { of processes }\end{array}$ & $\begin{array}{l}\text { No Eq. } \\
\text { text }\end{array}$ & in $\quad i$ & $f$ & $r$ & $S_{o}$ & Nos. of compounds in Table 1 \\
\hline $\begin{array}{l}C_{p} \\
\text { Stibium compounds }\end{array}$ & 30 & $117.2 \pm 32.5$ & $2.3 \pm 0.6$ & 0.973 & 27.4 & XV-XVII \\
\hline$\Delta_{\mathrm{vap}} H^{\circ}$ & 16 & $1.9 \pm 7.8$ & $1.2 \pm 0.1$ & 0.993 & 6.6 & XVIII-XX \\
\hline$\Delta_{\mathrm{c}} H^{\mathrm{o}}$ & 24 & $-528.6 \pm 34.6$ & $-107.8 \pm 0.6$ & 0.999 & 29.2 & XVIII-XX \\
\hline$\Delta_{\mathrm{f}} H_{\text {cond }}^{\mathrm{o}}$ & 25 & $-155.5 \pm 80.0$ & $5.3 \pm 1.4$ & 0.967 & 67.4 & XVIII-XX \\
\hline $\begin{array}{l}\triangle_{\mathrm{f}} H^{\circ} \text { gas } \\
\text { Bismuth compounds }\end{array}$ & 26 & $-152.6 \pm 88.4$ & $6.5 \pm 1.5$ & 0.973 & 74.6 & XVIII-XX \\
\hline$\Delta_{\text {vap }} H^{\circ}$ & 17 & $5.3 \pm 9.8$ & $1.2 \pm 0.2$ & 0.990 & 8.2 & XXI-XXIII \\
\hline$\Delta_{\mathrm{c}} H^{\mathrm{o}}$ & 27 & $-621.4 \pm 113.2$ & $-106.6 \pm 2.0$ & 0.999 & 95.4 & XXI-XXIII \\
\hline$\Delta_{\mathrm{f}} H_{\text {cond }}^{\mathrm{o}}$ & 28 & $14.5 \pm 68.4$ & $5.0 \pm 1.2$ & 0.973 & 57.7 & XXI-XXIII \\
\hline $\begin{array}{l}\Delta_{\mathrm{f}} H_{\text {gas }}^{\mathrm{o}} \\
\text { Oxygen compounds }\end{array}$ & 29 & $18.6 \pm 77.3$ & $6.2 \pm 1.3$ & 0.977 & 65.2 & XXI-XXIII \\
\hline$\Delta_{\mathrm{c}} G^{\mathrm{o}}$ & 46 & $-143.0 \pm 29.3$ & $-105.2 \pm 0.8$ & 0.999 & 26.7 & XXIV-XXVII, XXXI \\
\hline$\Delta_{\mathrm{f}} G_{\text {cond }}^{\mathrm{o}}$ & 47 & $-194.5+55.2$ & $-5.9+1.9$ & 0.909 & 38.2 & XXIV- XXVII \\
\hline$\Delta_{\mathrm{vap}} H^{\circ}$ & 31 & $14.5 \pm 3.0$ & $0.5 \pm 0.1$ & 0.953 & 2.6 & XXIV-XXXI \\
\hline$\Delta_{\mathrm{c}} H^{\mathrm{o}}$ & 35 & $-180.0 \pm 28.4$ & $-106.8 \pm 0.7$ & 0.999 & 26.9 & XXIV-XXXI \\
\hline$\Delta_{\mathrm{f}} H_{\text {cond }}^{\mathrm{o}}$ & 37 & $-157.9 \pm 24.1$ & $-4.9 \pm 0.6$ & 0.973 & 12.9 & XXV-XXX \\
\hline$\Delta_{\mathrm{f}} H_{\text {gas }}^{\mathrm{o}}$ & 38 & $-137.4 \pm 15.7$ & $-4.5 \pm 0.4$ & 0.980 & 14.0 & XXIV-XXX \\
\hline$S_{\text {cond }}^{o}$ & 41 & $73.3 \pm 24.5$ & $6.8 \pm 0.8$ & 0.985 & 16.9 & XXIV-XXX \\
\hline$\Delta_{\mathrm{c}} S_{\text {cond }}^{\mathrm{o}}$ & 45 & $29.6 \pm 44.9$ & $-11.3 \pm 1.5$ & 0.981 & 31.0 & XXIV-XXVII \\
\hline $\begin{array}{l}C_{p} \\
\text { Sufur compounds }\end{array}$ & 42 & $59.1 \pm 6.4$ & $4.7 \pm 0.2$ & 0.997 & 5.5 & XXIV-XXVIII, XXX \\
\hline$\Delta_{\mathrm{c}} G^{\mathrm{o}}$ & 49 & $-832.7 \pm 3.3$ & $-106.1 \pm 0.1$ & 0.999 & 1.3 & XXXIV, XXXV, XXXVII-XL \\
\hline$\Delta_{\mathrm{f}} G_{\text {cond }}^{\mathrm{o}}$ & 50 & $-58.5 \pm 5.4$ & $-5.9 \pm 0.2$ & 0.998 & 2.1 & XXXIV, XXXV, XXXVII-XL \\
\hline$\Delta_{\mathrm{vap}} H^{\circ}$ & 32 & $14.7 \pm 2.6$ & $0.9 \pm 0.1$ & 0.964 & 3.1 & XXXII-XLI \\
\hline$\Delta_{\mathrm{c}} H^{\mathrm{o}}$ & 36 & $-876.9 \pm 5.1$ & $-108.7 \pm 0.2$ & 0.999 & 4.1 & XXXII-XXXV, XXXVII-XL \\
\hline$\Delta_{\mathrm{f}} H_{\text {cond }}^{\mathrm{o}}$ & 39 & $-11.4 \pm 5.4$ & $-4.5 \pm 0.2$ & 0.994 & 4.4 & XXXII-XXXV, XXXVII-XL \\
\hline$\Delta_{\mathrm{f}} H_{\text {gas }}^{\mathrm{o}}$ & 40 & $9.8 \pm 7.5$ & $-3.9 \pm 0.3$ & 0.985 & 6.1 & XXX-XXXV, XXVII-XL \\
\hline$S_{\text {cond }}^{o}$ & 43 & $153.6 \pm 23.6$ & $4.9 \pm 0.8$ & 0.940 & 10.5 & XXXIV, XXXV-XL \\
\hline$\Delta_{\mathrm{c}} S_{\text {cond }}^{\mathrm{o}}$ & 48 & $-140.9 \pm 29.8$ & $-8.9 \pm 1.0$ & 0.977 & 11.8 & $\begin{array}{l}\text { XXXIV, XXXV, XXXVI, } \\
\text { XXXVII-XL }\end{array}$ \\
\hline $\begin{array}{l}C_{p} \\
\text { Fluor compounds }\end{array}$ & 44 & $100.8 \pm 11.4$ & $3.2 \pm 0.3$ & 0.969 & 9.5 & XXXIV-XLI \\
\hline$\Delta_{\text {vap }} H^{\circ}$ & 57 & $15.5 \pm 1.2$ & $0.8 \pm 0.04$ & 0.995 & 0.7 & XLII-XLIX \\
\hline$\Delta_{\mathrm{c}} H^{\mathrm{o}}$ & 58 & $-135.3 \pm 202.1$ & $-116.5 \pm 6.8$ & 0.990 & 192.5 & XLII-XLIX \\
\hline$\Delta_{\mathrm{f}} H_{\text {gas }}^{\mathrm{o}}$ & 59 & $-619.8 \pm 72.8$ & $13.4 \pm 3.0$ & 0.933 & 51.6 & XLIII- XLVI, XLIX \\
\hline$S_{\text {cond }}^{0}$ & 60 & $94.2 \pm 29.2$ & $4.8 \pm 1.1$ & 0.952 & 4.8 & XLII, XLIII, XLV, XLVI \\
\hline $\begin{array}{l}C_{p} \\
\text { Chlor compounds }\end{array}$ & 61 & $56.2 \pm 15.6$ & $3.9 \pm 0.6$ & 0.967 & 2.6 & XLII-XLVI \\
\hline$\Delta_{\text {vap }} H^{\circ}$ & 62 & $15.9 \pm 2.3$ & $0.8 \pm 0.1$ & 0.964 & 2.4 & L-LVIII \\
\hline$\Delta_{\mathrm{c}} H^{\mathrm{o}}$ & 63 & $-498.4 \pm 12.4$ & $-109.1 \pm 0.5$ & 0.999 & 13.0 & L-LVI, LXVIII \\
\hline$\Delta_{\mathrm{f}} H_{\text {cond }}^{\mathrm{o}}$ & 64 & $-122.4 \pm 23.3$ & $-3.6 \pm 0.8$ & 0.931 & 17.3 & LIII-LVI, LVIII \\
\hline$\Delta_{\mathrm{f}} H_{\text {gas }}^{\circ}$ & 65 & $-100.1 \pm 14.7$ & $-3.0 \pm 0.6$ & 0.915 & 15.2 & LI-LVI, LVIII \\
\hline $\begin{array}{l}C_{p} \\
\text { Brom compounds }\end{array}$ & 66 & $60.9 \pm 12.2$ & $4.8 \pm 0.5$ & 0.977 & 11.3 & L, LI, LIII-LVII \\
\hline$\Delta_{\text {vap }} H^{\circ}$ & 67 & $20.4 \pm 1.9$ & $0.8 \pm 0.1$ & 0.970 & 2.3 & LIX-LXVI \\
\hline$\Delta_{\mathrm{c}} H^{\mathrm{o}}$ & 68 & $-520.6 \pm 16.7$ & $-109.2 \pm 0.7$ & 0.999 & 16.7 & LIX, LXI-LXIII, LXV, LXVI \\
\hline$\Delta_{\mathrm{f}} H^{\mathrm{o}}$ cond & 69 & $-73.8 \pm 4.5$ & $-3.9 \pm 0.2$ & 0.993 & 5.6 & LX-LXVI \\
\hline$\Delta_{\mathrm{f}} H_{\text {gas }}^{\circ}$ & 70 & $-41.0 \pm 6.1$ & $-3.4 \pm 0.3$ & 0.988 & 7.4 & LX-LXIII, LXV, LXIV \\
\hline $\begin{array}{l}C_{p} \\
\text { Iod compounds }\end{array}$ & 71 & $70.1 \pm 2.6$ & $4.6 \pm 0.1$ & 0.999 & 0.9 & LXI-LXIV \\
\hline$\Delta_{\mathrm{vap}} H^{\mathrm{o}}$ & 72 & $22.4 \pm 2.3$ & $1.0 \pm 0.1$ & 0.987 & 1.8 & LXVII-LXXII \\
\hline$\Delta_{\mathrm{c}} H^{\mathrm{o}}$ & 73 & $-603.0 \pm 9.8$ & $107.6 \pm 0.4$ & 0.999 & 7.3 & LXVII-LXX, LXXII \\
\hline$\Delta_{\mathrm{f}} H_{\text {cond }}^{\mathrm{o}}$ & 74 & $-79.9 \pm 11.5$ & $5.1 \pm 0.5$ & 0.996 & 8.3 & LXVIII-LXX \\
\hline$\Delta_{\mathrm{f}} H_{\text {gas }}^{o}$ & 75 & $-56.2 \pm 10.8$ & $6.1+0.4$ & 0.997 & 7.8 & LXVIII-LXX \\
\hline$C_{p}$ & 76 & $85.2 \pm 22.4$ & $3.5 \pm 1.2$ & 0.943 & 14.1 & LXVII, LXX, LXXI \\
\hline
\end{tabular}

$\Delta_{\text {vap }} H^{\mathrm{o}}=(14.5 \pm 3.0)+(0.5 \pm 0.1)(N-h g) \quad r 0.953, S_{\mathrm{o}} 2.6, n 8$ (compounds XXIV-XXXI)

$\Delta_{\text {vap }} H^{0}=(14.7 \pm 2.6)+(0.9 \pm 0.1)(N-h g) \quad r 0.964, S_{\mathrm{o}} 3.1, n 10$ (compounds XXXII-XLI)

The processes of combustion of both types the given above compounds are characterized by schemes (33 и and 34)

$$
\begin{aligned}
& \mathrm{R}_{2} \mathrm{O}+n \mathrm{O}_{2} \rightarrow x \mathrm{CO}_{2} \text { (gas) }+y \mathrm{H}_{2} \mathrm{O} \text { (liq) } \\
& \mathrm{R}_{2} \mathrm{~S}+n \mathrm{O} 2 \rightarrow x \mathrm{CO}_{2} \text { (gas) }+y \mathrm{H}_{2} \mathrm{O}\left(\text { liq) }+z \mathrm{SO}_{2}\right.
\end{aligned}
$$

On the basis of known data on the heats of combustion of ethers and sulfides (Table 1) the equations (35 and 36) have been received (Table 2) 
$\Delta_{\mathrm{c}} H^{\mathrm{o}}=(-180.0 \pm 28.4)-(106.8 \pm 0.7)(N-h g) r 0.999, S_{\mathrm{o}} 26.9, n 8$ (compounds XXIV-XXXI)

$\Delta_{\mathrm{c}} H^{\mathrm{o}}=(-876.9 \pm 5.1)-(108.7 \pm 0.2)(N-h g) r 0.999, S_{\mathrm{o}} 4.1, n 9$ (compounds XXXII-XXXV, XXXVII-XL)

The same conclusion can be made concerning the heats of formation of ethers (Eqs. 37 and 38) and sulfides (Eqs.

$$
\begin{aligned}
& \Delta_{\mathrm{f}} H_{\text {cond }}^{\mathrm{o}}=(-157.9 \pm 24.1)-(4.9 \pm 0.6)(N-h g) \quad r 0.973, S_{\mathrm{o}} 12.9, n 6 \text { (compounds XXV-XXX) } \\
& \Delta_{\mathrm{f}} H_{\text {gas }}^{\mathrm{o}}=(-137.4 \pm 15.7)-(4.5 \pm 0.4)(N-h g) \quad r 0.980, S_{\mathrm{o}} 14.0, n 7 \text { (compounds XXIV-XXX) } \\
& \Delta_{\mathrm{f}} H_{\text {cond }}^{\mathrm{o}}=(-11.4 \pm 5.4)-(4.5 \pm 0.2)(N-h g) \quad r 0.994, S_{\mathrm{o}} 4.4, n 8 \text { (compounds XXXII-XXXV, XXXVII-XL) } \\
& \Delta_{\mathrm{f}} H_{\text {gas }}^{\mathrm{o}}=(9.8 \pm 7.5)-(3.9 \pm 0.3)(N-h g) \quad r 0.985, S_{\mathrm{o}} 6.1, n 8 \text { (compounds XXXII-XXXV, XXXVII-XL) }
\end{aligned}
$$

The experimental values of entropy of formation and literature (Table 1), that has enabled to calculate the heat capacity in condensed phase for ethers (XXIV-XXVIII, equations (41 and 42) for ethers and the equations (43 and $\mathrm{XXX}, \mathrm{XXXI})$ and sulfides (XXXIV-XLI) are known in the 44) for sulfides.

$$
\begin{aligned}
& S_{\text {cond }}^{\mathrm{o}}=(73.3 \pm 24.5)+(6.8 \pm 0.8)(N-h g) \quad r 0.985, S_{\mathrm{o}} 16.9, n 4 \text { (compounds XXIV-XXVII) } \\
& C_{p}=(59.1 \pm 6.4)+(4.7 \pm 0.2)(N-h g) \quad r 0.997, S_{\mathrm{o}} 5.5, n 6 \text { (compounds XXIV-XXVIII, XXX) } \\
& S_{\text {cond }}^{\mathrm{o}}=(153.6 \pm 23.5)+(4.9 \pm 0.8)(N-h g) r 0.940, S_{\mathrm{o}} 10.5, n 7 \text { (compounds XXXIV-XL) } \\
& C_{p}=(100.8 \pm 11.4)+(3.2 \pm 0.3)(N-h g) \quad r 0.969, S_{0} 9.5, n 8 \text { (compounds XXXIV-XLI) }
\end{aligned}
$$

With the use of Eqs. (12-14) the entropies of combustion, free energies of combustion and formation in condensed state of ethers and sulfides have been calculated (Table 1) and have allowed to produce the Eqs. (45-50, Table 2) correspondingly.

$$
\begin{aligned}
& \Delta_{\mathrm{c}} S_{\text {cond }}^{\mathrm{o}}=(29.6 \pm 44.9)-(11.3 \pm 1.5)(N-h g) \quad r 0.981, S_{\mathrm{o}} 31.0, n 4 \text { (compounds XXIV-XXVII) } \\
& \Delta_{\mathrm{c}} G^{\mathrm{o}}=(-143.0 \pm 29.3)-(105.2 \pm 0.8)(N-h g) \quad r 0.999, S_{\mathrm{o}} 26.7, n 5 \text { (compounds XXIV-XXVII, XXXI) } \\
& \Delta_{\mathrm{f}} G_{\text {cond }}^{\mathrm{o}}=(-194.5 \pm 55.2)-(5.9 \pm 1.9)(N-h g) \quad r 0.909, S_{\mathrm{o}} 38.2, n 4 \text { (compounds XXIV-XXVII) } \\
& \Delta_{c} S_{\text {cond }}^{o}=(-140.9 \pm 29.8)-(8.9 \pm 1.0)(N-h g) \quad r 0.977, S_{0} 11.8, n 4 \text { (compounds XXIV, XXXV, XXXVI, XXXVII-XL) (48) } \\
& \Delta_{\mathrm{c}} G^{\mathrm{o}}=(-832.7 \pm 3.3)-(106.1 \pm 0.1)(N-h g) r 0.999, S_{\mathrm{o}} 1.3, n 6 \text { (compounds XXXIV, XXXV, XXXVII-XL) } \\
& \Delta_{\mathrm{f}} G_{\text {cond }}^{\mathrm{o}}=(-58.5 \pm 5.4)-(5.9 \pm 0.2)(N-h g) \quad r 0.998, S_{\mathrm{o}} 2.1, n 6 \text { (compounds XXXIV, XXXV, XXXVII-XL) }
\end{aligned}
$$

Necessary to add, that the calculated thermodynamic functions are in a good dependencies among themselves: for ethers it Eqs. (51-53), and for sulfides it (54-56).

$$
\begin{aligned}
& \Delta_{\mathrm{f}} H_{\text {cond }}^{\mathrm{o}}=(-11.4 \pm 5.4)-(4.5 \pm 0.2) S_{\text {cond }}^{\mathrm{o}} \quad r 0.949, S_{\mathrm{o}} 23.6, n 4 \text { (compounds XXIV-XXVII) } \\
& S_{\text {cond }}^{\mathrm{o}}=(100.5 \pm 40.9)-(0.6 \pm 0.1) C_{p} \quad r 0.946, S_{\mathrm{o}} 31.4, n 4 \text { (compounds XXIV-XXVII) } \\
& \Delta_{\mathrm{c}} H^{\mathrm{o}}=(-547.2 \pm 362.7)+(9.1 \pm 1.2) \Delta_{\mathrm{c}} S_{\text {cond }}^{\mathrm{o}} r 0.982, S_{\mathrm{o}} 279.2, n 4 \text { (compounds XXIV-XXVII) } \\
& \Delta_{\mathrm{f}} H_{\text {cond }}^{\mathrm{o}}=(44.1 \pm 25.7)-(0.6 \pm 0.1) S_{\text {cond }}^{\mathrm{o}} \quad r 0.972, S_{\mathrm{o}} 5.4, n 6 \text { (compounds XXXIV, XXXV, XXXVII-XL) } \\
& \Delta_{\mathrm{c}} H^{\mathrm{o}}=(615.7 \pm 506.7)+(11.6 \pm 1.2) \Delta_{\mathrm{c}} S_{\text {cond }}^{\mathrm{o}} r 0.978, S_{\mathrm{o}} 135.3, n 6 \text { (compounds XXXIV, XXXV, XXXVII-XL)(55) } \\
& S_{\text {cond }}^{\mathrm{o}}=(100.0 \pm 39.3)+(1.0 \pm 0.2) C_{p} \quad r 0.914, S_{\mathrm{o}} 12.5, n 7 \text { (compounds XXXIV-XL) }
\end{aligned}
$$

\subsection{Organic Compounds of VII ${ }^{\text {th }}$ Group (Fluorides, Bromides and Iodides )}

The thermodynamic parameters (the heats of vaporization, combustion, formation, entropy of formation and heat capacities) of halogen-containing organic compounds can be used in the calculations relatively to the number of their valence electrons only. Such situation has turned out, as processes of combustion of fluorides, bromines and iodides the various authors characterize differently: with formation water-containing $\mathrm{HF}, \mathrm{HCl}$ and $\mathrm{HBr}$ of a various structure or gaseous $\mathrm{HI}$ and crystalline $\mathrm{I}_{2}$ [9]. It does not give an opportunity to make calculations of entropy of combustion and free energies, as in the literature there are no data on entropy of formation the corresponding combustion products, which are necessary for the equation (12). 
For this reason the calculations of the heats of vaporization, combustion, formation, entropy of formation

For fluorides:

$$
\begin{array}{ll}
\Delta_{\text {vap }} H^{\mathrm{o}}=(15.5 \pm 1.2)+(0.8 \pm 0.04)(N-h g) & r 0.995, S_{\mathrm{o}} 0.7, n 6 \text { (compounds XLII-XLIX) } \\
\Delta_{\mathrm{c}} H^{\mathrm{o}}=(-135.3 \pm 202.1)-(116.5 \pm 6.8)(N-h g) & r 0.990, S_{\mathrm{o}} 192.5, n 8 \text { (compounds XLII-XLXIX) } \\
\Delta_{\mathrm{f}} H_{\mathrm{gas}}^{\mathrm{o}}=(-619.8 \pm 72.8)+(13.4 \pm 3.0)(N-h g) & r 0.933, S_{\mathrm{o}} 51.6, n 5 \text { (compounds XLIII-XLVI, XLXIX) } \\
S_{\text {cond }}^{\mathrm{o}}=(94.2 \pm 29.2)+(4.8 \pm 1.1)(N-h g) & r 0.952, S_{\mathrm{o}} 4.8, n 4 \text { (compounds XLII, XLIII, XLV, XLVI) } \\
C_{p}=(56.2 \pm 15.6)+(3.9 \pm 0.6)(N-h g) & r 0.967, S_{\mathrm{o}} 2.6, n 5 \text { (compounds XLII-XLVI) }
\end{array}
$$

For chlorides:

$$
\begin{array}{ll}
\Delta_{\text {vap }} H^{\mathrm{o}}=(15.9 \pm 2.3)+(0.8 \pm 0.1)(N-h g) & r 0.964, S_{\mathrm{o}} 2.4, n 9 \text { (compounds L-LVIII) } \\
\Delta_{\mathrm{c}} H^{\mathrm{o}}=(-498.4 \pm 12.4)-(109.1 \pm 0.5)(N-h g) & r 0.999, S_{\mathrm{o}} 13.0, n 8 \text { (compounds L-LVI, LXVIII) } \\
\Delta_{\mathrm{f}} H^{\mathrm{o}}{ }_{\text {cond }}=(-122.4 \pm 23.3)-(3.6 \pm 0.8)(N-h g) & r 0.931, S_{\mathrm{o}} 17.3, n 5 \text { (compounds LIII-LVI, LVIII) } \\
\Delta_{\mathrm{f}} H_{\text {gas }}^{\mathrm{o}}=(-100.1 \pm 14.7)-(3.0 \pm 0.6)(N-h g) & r 0.915, S_{\mathrm{o}} 15.2, n 7 \text { (compounds LI-LVI, LVIII) } \\
C_{p}=(60.9 \pm 12.2)+(4.8 \pm 0.5)(N-h g) & r 0.977, S_{\mathrm{o}} 11.3, n 7 \text { (compounds L, LI, LIII-LVII) }
\end{array}
$$

For bromines:

$$
\begin{aligned}
& \Delta_{\text {vap }} H^{\mathrm{o}}=(20.4 \pm 1.9)+(0.8 \pm 0.1)(N-h g) \quad r 0.970, S_{\mathrm{o}} 2.3, n 8 \text { (compounds LIX-LXVI) } \\
& \Delta_{\mathrm{c}} H^{\mathrm{o}}=(-520.6 \pm 1.6)-(109.2 \pm 0.7)(N-h g) r 0.999, S_{\mathrm{o}} 16.7, n 6 \text { (compounds LIX, LXI-LXIII, LXV, LXVI) } \\
& \Delta_{\mathrm{f}} H_{\text {cond }}^{\mathrm{o}}=(-73.8 \pm 4.5)-(3.9 \pm 0.2)(N-h g) \quad r 0.993, S_{\mathrm{o}} 5.6, n 7 \text { (compounds LX-LXVI) } \\
& \Delta_{\mathrm{f}} H_{\text {gas }}^{\mathrm{o}}=(-41.0 \pm 6.1)-(3.4 \pm 0.3)(N-h g) \quad r 0.988, S_{\mathrm{o}} 7.4, n 6 \text { (compounds LX-LXIII, LXV, LXVI) } \\
& C_{p}=(70.1 \pm 2.6)+(4.6 \pm 0.1)(N-h g) \quad r 0.999, S_{\mathrm{o}} 0.9, n 4 \text { (compounds LXI-LXIV) }
\end{aligned}
$$

For iodines:

$$
\begin{array}{ll}
\Delta_{\text {vap }} H^{\mathrm{o}}=(22.4 \pm 2.3)+(1.0 \pm 0.1)(N-h g) & r 0.987, S_{\mathrm{o}} 1.8, n 6 \text { (compounds LXVII-LXXII) } \\
\Delta_{\mathrm{c}} H^{\mathrm{o}}=(-603.0 \pm 9.8)-(107.6 \pm 0.4)(N-h g) & r 0.999, S_{\mathrm{o}} 7.3, n 5 \text { (compounds LXVII-LXX, LXXII) } \\
\Delta_{\mathrm{f}} H_{\text {cond }}^{\mathrm{o}}=(-79.9 \pm 11.5)+(5.1 \pm 0.5)(N-h g) & r 0.996, S_{\mathrm{o}} 7.8, n 3 \text { (compounds LXVIII-LXX) } \\
\Delta_{\mathrm{f}} H_{\text {gas }}^{\mathrm{o}}=(-56.2 \pm 10.8)+(6.1 \pm 0.4)(N-h g) & r 0.997, S_{\mathrm{o}} 7.8, n 3 \text { (compounds LXVIII-LXX) } \\
C_{p}=(85.2 \pm 22.4)+(3.4 \pm 1.2)(N-h g) & r 0.943, S_{\mathrm{o}} 14.1, n 3 \text { (compounds LXVII, LXX, LXXI) }
\end{array}
$$

The available thermodynamic data have allowed to receiving one dependence (77) for fluorine

$$
S_{\text {cond }}^{\mathrm{o}}=(22.1 \pm 10.2)+(1.2 \pm 0.1) C_{p} \quad r 0.997, S_{\mathrm{o}} 1.1, n 4 \text { (compounds XLII, XLIII, XLV, XLVI) }
$$

\section{Conclusion}

The analysis of thermodynamic functions and heat capacities of organic compounds of V-VII groups of Mendeleev's Periodic system allows making a some essential conclusions.

- The most significant conclusion is the successful application of the equation (1) for an estimation of the interrelation of all functions with a number of valence electrons without taking into account of lone electrons pairs.

- The stoichiometric coefficients, which have been deduced for the vaporization processes and heat capacities, to characterize difficultly enough. It is necessary to note only, that in all cases they reflect partially a various spatial structure of the investigated compounds.

- The contributions from free energy and entropy of combustion and formation are calculated only for oxygen, sulfur and partly fluorine compounds. For this reason their analysis causes the certain difficulties. Analyzing the data of Table 2 it is possible to add only, that $f$ coefficients in equation (1) for free energies of combustion are very close to such coefficients for the heat of combustion processes.

- It has been found, that calculated thermodynamic functions of ethers and sulfides are in good 
interdependence from each other: $\Delta_{\mathrm{c}} H^{0}-\Delta_{\mathrm{c}} S_{\text {cond }}^{\mathrm{o}}, \Delta H_{\mathrm{f}}^{\mathrm{o}}-$ $S_{\text {cond }}^{\mathrm{o}}, S_{\text {cond }}^{\mathrm{o}}-C_{p}$.

\section{References}

[1] Ovchinnikov V.V., Thermochemistry of heteroatomic compounds: analysis and calculation of thermodynamic functions of organometallic compounds of I-IV groups of Mendeleev's Periodic table, AJPC, 2, 52-59 (2013).

[2] Ovchinnikov V.V., Thermochemistry of heteroatomic compounds: Thermochemistry of Heteroatomic Compounds: Interdependence between of some thermochemical Parameters of the different Classes organic nitro compounds and a Number of valence Electrons in their Molecules, ACSJ, 3, 11-23 (2013).

[3] Ovchinnikov V.V., Thermochemistry of Heteroatomic Compounds: Analysis and Calculation of Some Thermodynamic Functions of Saturated Alkanes, ACSJ, in press (2013).

[4] Ovchinnikov V.V., Thermochemistry of Heteroatomic Compound: Some thermodyna- mic Aspects of Combustion and Formation of Carbohydrates of Different Structures, Chem. Journal. 2, 2, 59-65 (2013).

[5] Ovchinnikov V.V., Thermochemistry of heteroatomic compounds: analysis and calculation of thermodynamic functions of amino acids and some peptides of different space structure, AJPC, 1, 1, 8-15 (2013).

[6] Kharasch M.S. and Sher B. The electronic conception of valence and heats of combustion of organic compounds, J. Phys. Chem., 25, 625-658(1925).

[7] Ovchinnikov V.V., Thermochemistry of Heteroatomic Compounds: Enthalpy of Combustion of Organic Compounds of Group I-VII Elements, Doklady Physical Chemistry, 443, 49-52 (2012).

[8] Karapetyanz M.Kh. and Karapetyanz M.L., The basic thermodynamic constants of inorganic and organic chemistry, 471 pp. (1968).

[9] Cox J.D. and Pilcher G.,,Thermochemistry of Organic and Organometallic Compounds. Academic Press - London and New York, 640 pp. (1970)

[10] Issoire J. and Long, C., Etude de la thermodynamique chimique de la reaction de formation des methylamines, Bull. Soc. Chim. France, 2004-2012 (1960).

[11] Muller J.-A., Sur les chaleurs de combustion et les poids spécifiques des methylamines, Ann. Chim. Phys., 20, 116130 (1910).

[12] Aston J.G., Sagenkahn M.L., Szasa G.J., Moessen G.W. and Zuhr H.F., The heat capacity and entropy, heats of fusion and vaporization and the vapor pressure of trimethylamine. The entropy from spectroscopic and molecular data, J. Am. Chem. Soc., 66, 1171-1177 (1944).

[13] Wadso I., Enthalpies of vaporization of organic compounds, Acta Chem. Scand., 23, 2061 (1969).

[14] Lebedeva N.D., Heats of combustion and formation of aliphatic tertiary amine homologues, Russ. J. Phys. Chem. (Engl. Transl.), 40, 1465-1467 (1966).
[15] Steele W.V., Chirico R.D., Knipmeyer S.E., Nguyen A., Smith N.K. and Tasker, I.R., Thermodynamic properties and ideal-gas enthalpies of formation for cyclohexene, phthalan (2,5-dihydrobenzo-3,4-furan), isoxazole, octylamine, dioctylamine, trioctylamine, phenyl isocyanate, and 1,4,5,6tetrahydropyrimidine, J. Chem. Eng. Data, 41, 1269-1284 (1996).

[16] Steele W.V., The standard enthalpies of formation of the triphenyl compounds of the Group $\mathrm{V}$ elements. 1. Triphenylamine and the $\mathrm{Ph}-\mathrm{N}$ bond-dissociation energy, J. Chem. Thermodyn., 10, 441-444 (1978).

[17] Smith R.H. and Andrews D.H., Thermal energy studies. II. Phenyl derivatives of metals, J. Am. Chem. Soc., 53, 36613667 (1931).

[18] Ovchinnikov V.V., Khazieva L.R, Lapteva L.I. and Konovalov A.I., Thermochemistry of heteroatomic compounds. 17. Theoretical calculation of enthalpy of vaporization of alkyl- and alkyl(aryl)phosphines, Russ. Chem. Bulletin, Int. Ed., 49, 32-37 (2000).

[19] Nistratov V.P., Sheiman M.S., Rabinovich I.B., Vasil'ev V.G., Karataev E.N. and Feshchenko I.A., Heat capacity and thermodynamic functions of trimethylarsine, Zhur. Fiz. Khim., 62(8), 2219-2221 (1988).

[20] Maslova V.A. and Faminskaya L.A., Specific heat phase transitions of triethylarsine and triethylgallium, Tr. Khim. Khim. Tekhnol., (2), 51-53 (1972).

[21] Smith R.H. and Andrews D.H., Thermal energy studies. II. Phenyl derivatives of metals, J. Am. Chem. Soc., 53, 36613667 (1931).

[22] Ovchinnikov V.V., Makeeva T.B., Lapteva L.I., Valiullina V.A., Pilishkina L.M. and Konovalov A.I., Thermochemistry of heteroatomic compounds. Part 7. Enthalpies of solution, vaporization and solvation of three- coordinated arsenic derivatives, J. Thermal Analysis, 45, 735-739 (1995).

[23] Maslova V.A., Novoselova N.V., Moseeva E.M., Berezhnaya N.D. and Rabinovich I.B., Specific heat and phase changes of triethylindium, triethyl antimony, and trimethyl gallium, Trudy. Khim. Khim. Tekhnol., (2), 51-52 (1973).

[24] Nistratov V.P., Rabinovich I.B., Sheiman M.S., Smirnova N.N., Zelyaev I.A. and Feshchenko I.A., Specific heat and thermodyanamic characteristics of triethylbismuth, Zhur. Fiz. Khim., 63, 1779-1783 (1989).

[25] Kennedy R.M., Sagenkahn M. and Aston, J.G., The heat capacity and entropy, heats of fusion and vaporization, and the vapor pressure of dimethyl ether. The density of gaseous dimethyl ether, J. Am. Chem. Soc., 63, 2267- 2272 (1941).

[26] Pilcher G., Pell A.S. and Coleman D.J., Measurements of heats of combustion by flame calorimetry. Part 2-Dimethyl ether, methyl ethyl ether, methyl n-propyl ether, methyl isopropyl ether, Trans. Faraday Soc., 60, 499- 505 (1964).

[27] Majer V. and Svoboda V., Enthalpies of Vaporization of Organic Compounds: A Critical Review and Data Compilation, Blackwell Scientific Publications, Oxford, pp. 300 (1985).

[28] Murrin J.W. and Goldhagen S., Determination of the C-O bond energy from the heats of combustion of four aliphatic ethers, NAVORD Report No. 5491, U.S. Naval Powder Factory Res. \& Dev. Dept., 1-14 (1957). 
[29] Pihlaja K. and Heikkil J., Heats of combustion. Diethyl ether and 1,1-diethoxyethane, Acta Chem. Scand., 22, 27312732 (1968).

[30] Counsell J.F., Lee D.A. and Martin J.F., Thermodynamic properties of organic oxygen compounds: Part XXVI. Diethyl ether, J. Chem. Soc. A, 313-316 (1971).

[31] Majer V., Wagner Z., Svoboda V. and Cadek V., Enthalpies of vaporization and cohesive energies for a group of aliphatic ethers, J. Chem. Thermodyn., 12, 387-391 (1980).

[32] Colomina M., Pell A.S., Skinner H.A. and Coleman D.J., Heats of combustion of four dialkylethers, Trans. Faraday Soc., 61, 2641 (1965).

[33] Andon R.J.L., Thermodynamic properties of organic oxygen compounds. 39. Heat capacity of n-propyl ether, J. Chem. Thermodyn., 7, 587-592 (1975).

[34] Cobos J.C., Casanova C., Roux-Desgranges G. and Grolier J.-P.E., Excess properties of mixtures of some nalkoxyethanols with organic solvents. II. VEm and CEp,m with di- n-butylether at $298.15 \mathrm{~K}$, J. Chem. Thermodynam., 19, 791-796 (1987).

[35] Fenwick J.O., Harrop D. and Head A.J., Thermodynamic properties of organic oxygen compounds. 41. Enthalpies of formation of eight ethers, J. Chem. Thermodyn., 7, 943-954 (1975).

[36] Steele W.V., Chirico R.D., Knipmeyer S.E., Nguyen A. and Smith N.K., Thermodynamic properties and ideal-gas enthalpies of formation for butyl vinyl ether, 1,2dimethoxyethane, methylglycolate, bicyclo[2. 2.1]hept-2ene,5-vinylbicyclo- [2.2.1]hept-2- ene, trans-azobenzene, butyl acrylate, di-tert- butyl ether, and hexane-1,6-diol, J. Chem. Eng. Data, 41, 1285-1302 (1996).

[37] Morawetz E., Enthalpies of vaporization for a number of aromatic compounds, J. Chem. Thermodyn., 4, 455 (1972).

[38] Cass R.C., Fletcher S.E., Mortimer C.T., Springall H.D. and White T.R., Heats of combustion and molecular structure. Part V. The mean bond energy term for the $\mathrm{C}-\mathrm{O}$ bond in ethers, and the structures of some cyclic ethers, J. Chem. Soc., 1406-1410 (1958).

[39] Furukawa G.T., Ginnings D.C., McCoskey R.E. and Nelson R.A., Calorimetric properties of diphenyl ether from $0^{\circ}$ to $570^{\circ} \mathrm{K}$, J. Res. NBS, 46, 195-206 (1951).

[40] Voronkov M.G., Klyuchnikov V.A., Kolabin S.N., Shvets G.N., Varusin P.I., Deryagina E.N., Korchevin N.A. and Tsvetnitskaya S.I., Thermochemical properties of diorganyl chalcogenides and dichalcogenides $\mathrm{RMnR}(\mathrm{M}=\mathrm{S}, \mathrm{Se}, \mathrm{Te} ; \mathrm{n}$ $=1,2)$., Dokl. Phys. Chem. (Engl. Transl.), 307, 650-653 (1989).

[41] McCullough J.P., Hubbard W.N., Frow F.R., Hossenlopp I.A. and Waddington G., Ethanethiol and 2-thiapropane: Heats of formation and isomerization; the chemical thermodynamic properties from 0 to $1000^{\circ} \mathrm{K}, \mathrm{J}$. Am. Chem. Soc., 79, 561$566(1957)$

[42] Hubbard W.N. and Waddington G., The heat of combustion, formation and isomerization of propanethiol-1, propanethiol-2 and 2- thiabutane, Rec. Trav. Chim. Pays/Bas, 73, 910 (1954)

[43] Hubbard W.N., Good W.D. and Waddington G., The heats of combustion, formation and isomerization of the seven isomeric C4H10S alkane thiols and sulfides, J. Phys. Chem., 62, 614-617 (1958).

[44] Scott D.W., Finke H.L., Hubbard W.N., McCullough J.P., Oliver G.D., Gross M.E., Katz C., Williamson K.D., Waddington G. and Huffman H.M., 3-Thiapentane: heat capacity, heats of fusion and vaporization, vapor pressure, entropy, heat of formation and thermodynamic functions, J. Am. Chem. Soc., 74, 4656-4662 (1952).

[45] McCullough J.P., Finke H.L., Messerly J.F., Pennington R.E., Hossenlopp I.A. and Waddington G., 3-Methyl-2thiabutane: calorimetric studies from 12 to $500 \mathrm{~K}$., the chemical thermodynamic properties from 0 to $1000 \mathrm{~K}, \mathrm{~J}$. Am. Chem. Soc., 77, 6119-6125 (1955).

[46] Scott D.W., Finke H.L., McCullough J.P., Messerly J.F., Pennington R.E., Hossenlopp I.A. and Waddington G., 1Butanethiol and 2- thiapentane. Experimental thermodynamic studies between 12 and 500 'K., thermodynamic functions by a refined method of increments, J. Am. Chem. Soc., 79, 1062-1068 (1957).

[47] McCullough J.P., Finke H.L., Hubbard W.N., Todd S.S., Messerly J.F., Douslin D.R. and Waddington G., Thermodynamic properties of four linear thiaalkanes, J. Phys. Chem., 65, 784-791 (1961).

[48] Good W.D., Enthalpies of combustion of 18 organic sulfur compounds related to petroleum, J. Chem. Eng. Data, 17, 158-162 (1972).

[49] Messerly J.F., Todd S.S. and Guthrie G.B., Low Temperature Thermal Properties of Cyclohexanethiol and 2,4-Dimethyl3- thiapentane, J. Chem. Eng. Data, 12, 426-429 (1967).

[50] Mackle H. and Mayrick R.G., Studies in thermochemistry of organic sulphides. Part 3.- The gas-phase heats of formation of diphenyl sulphide, dibenzyl sulphide, diphenyl disulphide,4-thia-1-hexene and 4-thia-5,5dimethyl-1-hexene, Trans. Faraday Soc., 58, 238-243 (1962).

[51] Scott D.W., McCullough J.P., Good W.D., Messerly J.F., Pennington R.E., Kincheloe T.C., Hossenlopp I.A., Douslin D.R and Waddington G., Fluorobenzene: Thermodynamic properties in the solid, liquid and vapor states, a revised vibrational assignment, J. Am. Chem. Soc., 78, 5457-5463 (1956).

[52] Good W.D., Scott D.W. and Waddington G., Combustion calorimetry of organic fluorine compounds by a rotatingbomb method, J. Phys. Chem., 60, 1080-1089 (1956).

[53] Good W.D., Lacina J.L., Scott D.W. and McCullough J.P., Combustion calorimetry of organic fluorine compounds. The heats of combustion and formation of the difluorobenzenes, 4-fluorotoluene and $\mathrm{m}$ - trifluorotoluic acid, J. Phys. Chem., 66, 1529- 1532 (1962).

[54] Scott D.W., Messerly J.F., Todd S.S., Hossenlopp I.A., Douslin D.R. and McCullough J.P., 4-Fluorotoluene: Chemical thermodynamic properties, vibrational assignment, and internal rotation, J. Chem. Phys., 37, 867873 (1962)

[55] Messerly J.F. and Finke H.L., Hexafluoro- benzene and 1,3-difluorobenzene. Low- temperature calorimetric studies and chemical thermodynamic properties, J. Chem. Thermodynam., 2, 867-880 (1970). 
[56] Scott D.W., Messerly J.F., Todd S.S., Hossenlopp I.A., Osborn A. and McCullough J.P., 1,2-Difluorobenzene: Chemical thermodynamic properties and vibrational assignment, J. Chem. Phys., 38, 532-539 (1963).

[57] Swarts F., Etudes thermochimiques sur les combinaisons organiques fluorees, J. Chim. Phys., 17, 3-70 (1919).

[58] Kolesov V.P., Shtekher S.N., Martynov A.M. and Skuratov S.M., Standard enthalpy of formation of 1,1-difluoroethane, Russ. J. Phys. Chem. (Engl. Transl.), 42, 975-976 (1968).

[59] Wadso I., Heats of vaporization of organic compounds II. Chlorides, bromides, and iodides, Acta Chem. Scand., 22, 2438 (1968).

[60] Platonov V.A. and Simulin, Yu.N., Determination of the standard enthalpies of formation of polychlorobenzenes. III. The standard enthalpies of formation of mono- 1,2,4- and 1,3,5-tri-, and 1,2,3,4- and 1,2,3,5- tetrachlorobenzenes, Russ. J. Phys. Chem. (Engl. Transl.), 59, 179-181 (1985).

[61] Kolesov V.P., Tomareva E.M., Skuratov S.M. and Alekhin S.P., Calorimeter having a rotating bomb for determining heats of combustion of chlorinated organic compounds, Russ. J. Phys. Chem. (Engl. Transl.), 41, 817-820 (1967).

[62] Stull D.R., A semi-micro calorimeter for measuring heat capacities at low temperatures, J. Am. Chem. Soc., 59, 2726-2733 (1937).

[63] Shehatta I., Heat capacity at constant pressure of some halogen compounds, Thermochim. Acta, 213, 1-10 (1993).

[64] Smith L., Bjellerup L., Krook S. and Westermark H., Heats of combustion of organic chloro compounds determined by the "quartz wool" method, Acta Chem. Scand., 7, 65 (1953).

[65] Fletcher R.A. and Pilcher G., Measurements of heats of combustion by flame calorimetry. Part 7.-Chloromethane, chloroethane, 1- chloropropane, 2-chloropropane, Trans. Faraday Soc., 67, 3191-3201 (1971).

[66] Kurbatov V.Ya., Heat capacity of liquids. 2. Heat capacity and the temperature dependence of heat capacity from halogen derivatives of acylic hydrocarbons, Zh. Obshch. Kim., 18, 372-389 (1948)

[67] Mathews J.H. and Fehlandt P.R., The heats of vaporization of some organic compounds, J. Am. Chem. Soc., 53, 321232 (1931).

[68] Stridth G. and Sunner S., Enthalpies of formation of some 1chloroalkanes and the $\mathrm{CH} 2$-increment in the 1-chloroalkanes series, J. Chem. Thermodyn., 7, 161-168 (1975).

[69] He J., An X. and Hu R., Measurements of enthalpies of formation of 2-chlorobutane and 1,2-dichlorobutane in gaseous state, Acta Chim. Sin., 50, 961-966 (1992).

[70] Shehatta I., Heat capacity at constant pressure of some halogen compounds, Thermochim. Acta, 213, 1-10 (1993).

[71] Arnett E.M. and Pienta, N.J., Stabilities of carbonium ions in solution. 12. Heats of formation of alkyl chlorides as an entree to heats of solvation of aliphatic carbonium ions, J. Am. Chem. Soc., 102, 3329-3334 (1980).

[72] Howlett K.E., The use of equilibrium constants to calculate thermodynamic quantities. Part II, J. Chem. Soc., 1784-1789 (1955).

[73] An X.-W. and Guo D.-J., Formation enthalpies and nonbonding interactions of hexachloro- cyclohexanes, Thermochim. Acta, 253, 235- 242 (1995).

[74] Smith L. and Bjellerup L., Neue Versuche mit beweglicher calorimetrischer Bombe, Acta Chem. Scand., 1, 566-570 (1947).

[75] Chernick C.L., Skinner H.A. and Wadso, I., Thermochemistry of metallic alkyls. Part 7. The heat of formation of mercury diphenyl, and of mercury phenyl chloride, Trans. Faraday Soc., 52, 1088-1093 (1956).

[76] Masi J.F. and Scott R.B., Some thermodynamic properties of bromobenzene from 0 to $1500 \mathrm{~K}$, J. Res., NBS 79A, 619-628 (1975).

[77] Kudchadker S.A. and Kudchadker A.P., Ideal gas thermodynamic properties of selected bromoethanes and iodoethane, J. Phys. Chem. Ref. Data, 8, 519-526 (1979).

[78] Bjellerup L., On the accuracy of heat of combustion data obtained with a precision moving-bomb calorimetric method for organic bromine compounds, Acta Chem. Scand., 15, 121-140 (1961).

[79] Davies J., Lacher J.R. and Park J.D., Reaction heats of organic compounds. Part 4.-Heats of hydrogenation of nand iso-Propyl bromides and chlorides, Trans. Faraday Soc., 61, 2413- 2416 (1965)

[80] Mansson M., Sellers P., Stridh G. and Sunner S., Enthalpies of vaporization of some 1- substituted n-alkanes, J. Chem. Thermodyn., 9, 91 (1977).

[81] Smith L., Corrected heats of combustion of organic iodine compounds, Acta Chem. Scand., 10, 884-886 (1956).

[82] Holm T., Thermochemistry of Grignard reagents. Enthalpies of formation of alkylmagnesium bromides and of alkyl bromides, J. Organomet. Chem., 56, 87-93 (1973).

[83] Deese R.F., Jr., Thermal energy studies. IV. Comparison of continuous and discontinuous methods of measuring heat capacities. Heat capacities of some alphatic bromides, J. Am. Chem. Soc., 53, 3673-3683 (1931).

[84] Carson A.S., Laye P.G., Pedley J.B., Welsby A.M., Chickos J.S. and Hosseini S., The enthalpies of formation of iodoethane, 1,2- diiodoethane, 1,3-diiodopropane, and 1,4diiodobutane, J. Chem. Thermodyn., 26, 1103- 1109 (1994).

[85] Brennan D. and Ubbelohde A.R., A thermochemical evaluation of bond strengths in some carbon compounds. Part IV. Bond- strength differences based on the reaction: RI $+\mathrm{HI}=\mathrm{RH}+\mathrm{I} 2$, where $\mathrm{R}=$ p-methoxyphenyl and cyclohexyl, J. Chem. Soc., 3011-3016 (1956). 